\title{
Hepatocytes, Rather than Cholangiocytes, Can Be the Major Source of Primitive Ductules in the Chronically Injured Mouse Liver
}

\author{
Sayaka Sekiya* and Atsushi Suzuki*i
}

From the Division of Organogenesis and Regeneration,* Medical Institute of Bioregulation, Kyushu University, Fukuoka; and the Core Research for Evolutional Science and Technology (CREST), ${ }^{\dagger}$ Japan Science and Technology Agency, Kawaguchi, Japan

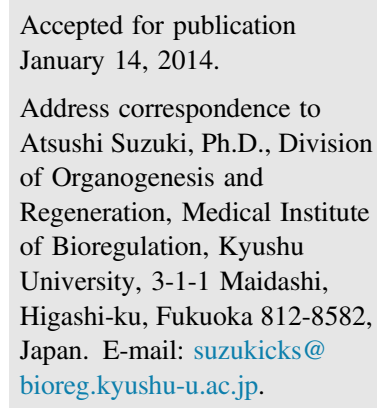

\begin{abstract}
The proliferation of biliary lineage cells in chronic liver diseases, which leads to formation of primitive ductules in portal areas of the hepatic lobule, may be important not only for liver regeneration, but also for initiation of liver cancer. Thus, understanding how these primitive ductular cells emerge and proliferate in chronically injured liver holds promise for development of therapeutic strategies for liver diseases. However, the origin of these primitive ductular cells remains controversial. Here, we use a method for genetic lineage tracing to determine the origin of cells that form primitive ductules in a mouse model of chronic liver injury. Our results show that hepatocytes, rather than cholangiocytes, are the major source of cells for the primitive ductules formed in response to chronic liver damage. Moreover, activation of the Notch-Hes1 signaling axis is important for conversion of hepatocytes into primitive ductular cells in chronically injured liver. These findings should be valuable in elucidating the mechanism of liver regeneration associated with the fate-conversion of hepatocytes and in developing therapeutic strategies for liver diseases. (Am J Pathol 2014, 184: 1468-1478; http://dx.doi.org/ 10.1016/j.ajpath.2014.01.005)
\end{abstract}

The small epithelial cells (often called oval cells) that are histologically identified as cells expressing biliary lineage markers and forming primitive ductules in portal areas of the hepatic lobule appear in response to several types of chronic injury in the human liver, including hepatitis $\mathrm{C}$ virus infection, hemochromatosis, and alcoholic liver disease. ${ }^{1}$ These liver pathologies are associated with an increased risk of primary liver cancers. ${ }^{2-4}$ To study such human diseases, experimental rodent models have been developed with chronic liver injury induced by potential carcinogens, including azo dyes, choline-deficient/ethionine-containing (CDE) diet, D-galactosamine, 2-acetylaminofluorene, 3,5diethoxycarbonyl-1,4-dihydrocollidine (DDC), and 1,4-bis $\left[N, N^{\prime}\right.$-di(ethylene)-phosphamide]-piperazine (dipin). ${ }^{5-10}$ In these models, biliary lineage cells that form primitive ductules appear and proliferate around the portal veins to regenerate damaged liver tissues. Moreover, deletion of Tp53 (alias p53) in these cells resulted in formation of malignant tumors on injection into immunodeficient mutant mice, suggesting that these rodent models of chronic liver injury mirror the progression of human liver pathologies. ${ }^{11,12}$ Thus, to develop therapeutic strategies for liver diseases, it is important to understand how biliary lineage cells emerge and form primitive ductules in models of chronic liver injury.

Previous studies involving histological observations of liver injury and carcinogenesis have suggested that the portal biliary ductules and the canals of Hering, through which the terminal segments of the biliary system connect with hepatocytes in

Supported in part by the Program for Improvement of the Research Environment for Young Researchers from the Special Coordination Funds for Promoting Science and Technology commissioned by the Ministry of Education, Culture, Sports, Science and Technology (MEXT) of Japan, MEXT Grants-in-Aid for Scientific Research, a Health Labour Sciences Research Grant in Japan, the Precursory Research for Embryonic Science and Technology Program of the Japan Science and Technology Agency, and the Core Research for Evolutional Science and Technology Program of the Japan Science and Technology Agency (all to A.S.).

Disclosures: None declared. 
liver parenchyma, constitute a niche for cells capable of generating biliary lineage cells that form primitive ductules. ${ }^{13,14}$ Recent evidence from Cre-based genetic lineagetracing experiments also showed that primitive ductular cells found in the mouse DDC and CDE models of chronic liver injury are, at least in part, derived from cholangiocytes lining the intrahepatic bile ducts. ${ }^{15-17}$ In addition to this biliary origin scenario, other histological and cell culture-based studies have suggested that mature hepatocytes can transdifferentiate into biliary lineage cells that form ductular structures, in which case hepatocytes could be a potential source of cells that also express biliary lineage markers and form primitive ductular structures in chronically injured liver. ${ }^{18-20}$ Moreover, a recent study using adeno-associated virus (AAV) with a thyroid binding globulin (TBG) promoterdriven Cre recombinase showed that hepatocytes can be converted into biliary lineage cells in DDC-induced chronic liver injury. ${ }^{21}$ In that study, however, the proportion of hepatocyte-derived cytokeratin 19 (CK19)-positive primitive ductular cells (indicative of terminal conversion of hepatocytes to biliary lineage cells) was only approximately $14 \%$; however, several biliary lineage markers were expressed in $30 \%$ to $50 \%$ of hepatocytes. Thus, it is difficult to conclude whether hepatocytes actively contribute to primitive ductule formation in DDC-treated chronically injured liver. Indeed, other hepatocyte fate-tracing studies using mice with chimeric livers or AAV with a transthyretin (Ttr) promoter-driven Cre recombinase have shown that the primitive ductular cells induced in the mouse DDC model were not derived from hepatocytes. ${ }^{22,23}$ Furthermore, although it has also been suggested that the primitive ductular cells found in chronic liver diseases originate from bone marrow-derived cells, ${ }^{24}$ evidence from a study combining bone marrow chimeras with the mouse DDC model indicated no contribution of bone marrow-derived cells to primitive ductule formation in liver. ${ }^{22}$

Overall, the cellular origin of the biliary lineage cells that appear and form primitive ductules in chronically injured liver remains controversial. In the present study, we performed Cre-based genetic lineage-tracing analyses to determine the fates of hepatocytes and cholangiocytes using more definitive markers for these cells and to identify which cell type is the source of the primitive ductular cells that appear in chronically injured liver. Specifically, hepatocytes expressing albumin or cholangiocytes expressing CK19 in liver were heritably labeled in mutant mice with a tamoxifen (TMX) inducible Cre/loxP system. The mutant mice were then fed a DDC-containing diet to induce chronic liver injury and primitive ductule formation. Our present findings show that hepatocytes, rather than cholangiocytes, give rise to the majority of the biliary lineage cells that form primitive ductules in chronically injured liver, through Notch-mediated cell lineage conversion. Thus, at least in the mouse DDC model, albumin-positive hepatocytes can be the major source of the biliary lineage cells that form primitive ductules around the portal veins with a small number of CK19 ${ }^{+}$ cholangiocyte-derived cells in chronically injured liver.

\section{Materials and Methods}

\section{Mice}

The mice used in this study were C57BL/6 mice (CLEA Japan, Fuji, Japan), Alb-CreER ${ }^{T 2}$ mice [a gift from Drs. Pierre Chambon and Daniel Metzger (Institute of Genetics and Molecular and Cellular Biology, Strasbourg, France)], CK19-CreER ${ }^{T 2}$ mice, ${ }^{25}$ R26R lacZlacZ mice (Jackson Laboratory, Bar Harbor, ME), $R 26 R^{Y F P / Y F P}$ mice [a gift from Dr. Frank Costantini (Columbia University, New York, $\mathrm{NY})$ ], $R 26 R^{\text {Notch/+ }}$ mice [a gift from Dr. Douglas A. Melton (Harvard University, Cambridge, MA)], and Hes $1^{f / f l}$ mice [a gift from Dr. Ryoichiro Kageyama (Kyoto University, Kyoto, Japan)].

\section{Chemical Administration}

For induction of Cre activity, 8- to 10 -week-old mice were given a single intraperitoneal injection of 6.5 to $8.0 \mathrm{mg}$ TMX per mouse (Sigma-Aldrich, St. Louis, MO) dissolved in olive oil (Nacalai Tesque, Kyoto, Japan) at a concentration of $50 \mathrm{mg} / \mathrm{mL}^{25}$ To induce chronic liver injury, mice were fed a diet containing 0.1\% DDC (Sigma-Aldrich).

\section{Immunostaining}

Liver tissues with yellow fluorescent protein (YFP) expression were fixed with $2 \%$ paraformaldehyde at $4{ }^{\circ} \mathrm{C}$ overnight. ${ }^{25}$ After treatment with $30 \%$ sucrose at $4^{\circ} \mathrm{C}$ overnight, the tissues were embedded in Tissue-Tek OCT optimal cutting temperature compound (Sakura Finetek, Torrance, CA; Tokyo, Japan), and frozen sections were prepared. ${ }^{25}$ Liver tissues without YFP expression were directly embedded in the OCT compound, and frozen sections of these tissues were initially fixed with $4 \%$ paraformaldehyde for 5 minutes and then fixed with methanol for 5 minutes at room temperature. ${ }^{25}$ For albumin staining, antigen retrieval was performed by microwave heating in $0.01 \mathrm{~mol} / \mathrm{L}$ citrate buffer ( $\mathrm{pH} \mathrm{6.0)}$. After a washing in PBS containing $0.1 \%$ Tween 20 , followed by blocking, the sections were incubated with the following primary antibodies: rat anti-Ep-CAM (1:200; eBioscience, San Diego, CA), rabbit anti- $\beta$-gal (1:2000; Abcam, Cambridge, MA), goat anti-albumin (1:200; Bethyl Laboratories, Montgomery, TX), rabbit anti-keratin (1:2000; Dako, Carpinteria, CA), mouse anti-Hnf4 $\alpha$ (1:200; Perseus Proteomics, Tokyo, Japan), rabbit anti-CK19 (1:2000; a rabbit anti-mouse CK19 antibody generated against the peptide HYNNLPTPKAI ${ }^{25}$; GeneNet, Fukuoka, Japan), goat anti-Trop2 (1:50; R\&D Systems), rabbit anti-Sox9 (1:1000; EMD Millipore, Billerica, MA), rabbit anti-Cre (1:2000; Covance, Princeton, NJ), and rat anti-A6 [1:50; a gift from Dr. Valentina M. Factor (National Cancer Institute, Bethesda, MD)]. After a washing, the sections were incubated with Alexa Fluor 488-conjugated and/or Alexa Fluor 555-conjugated secondary antibodies (1:200; Life Technologies, Carlsbad, CA) and DAPI. Quantitative 

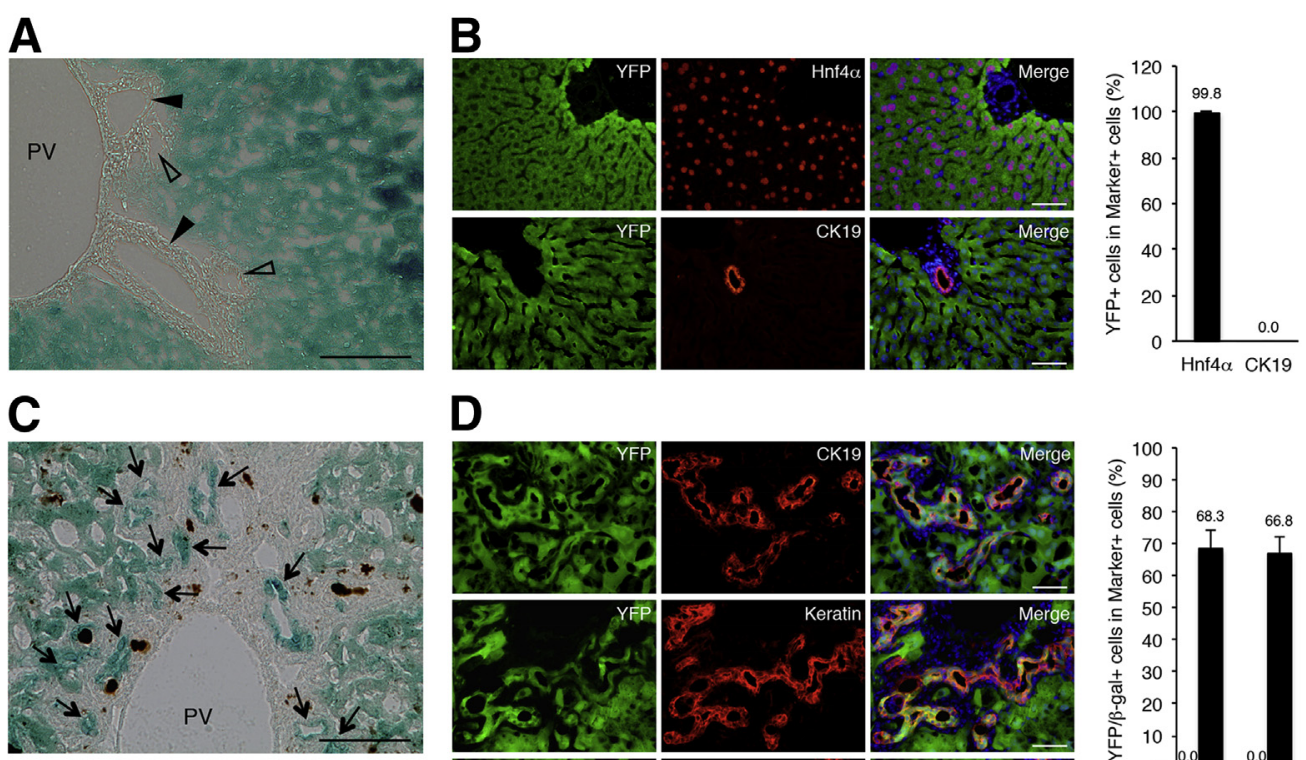

D
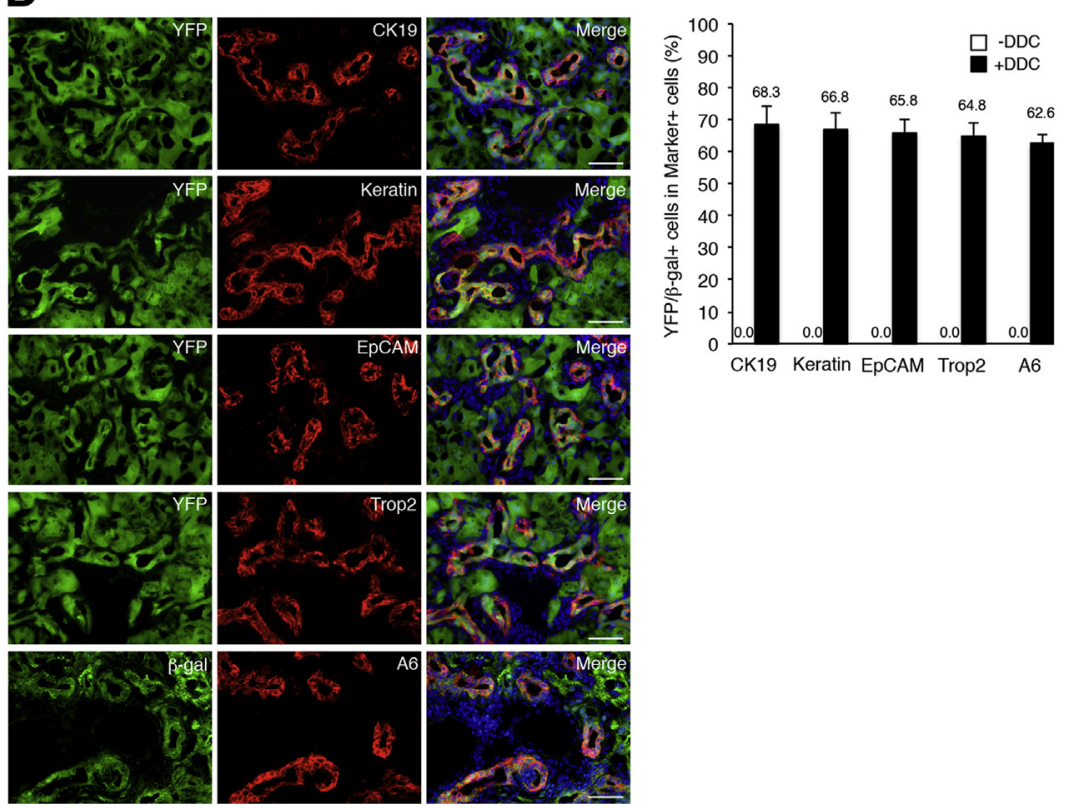

Figure 1 The majority of the primitive ductules that appear in chronically injured liver arise from hepatocytes. A: X-gal staining in liver of Alb$\mathrm{CreER}^{T 2} ; R 26 R^{\text {lacZ/+ }}$ mice fed a normal diet for 4 weeks after TMX injection. No lac $Z^{+}$cells are observed within intrahepatic bile ducts (black arrowheads) or blood vessels (PV and hepatic artery) (open arrowheads). B: Immunofluorescence staining for Hnf4 $\alpha$ and CK19 in liver of $A l b-C r e E R^{T 2} ; R 26 R^{Y F P /+}$ mice fed a normal diet for 4 weeks after TMX injection, with percentages of $\mathrm{Hnf}_{4} \alpha^{+}$and $\mathrm{CK} 19^{+}$cells that coexpress YFP. C: X-gal staining in liver of $A l b-C r e E R^{T 2} ; R 26 R^{\text {lacZ/ }+}$ mice fed a normal diet for 1 week after TMX injection and then a DDC-containing diet for 3 weeks. The primitive ductules (arrows) consist of lacZ ${ }^{+}$cells. D: Immunofluorescence staining of CK19, keratin, Ep-CAM, and Trop2 in liver of $A l b-C r e E R^{T 2} ; R 26 R^{Y F P /+}$ mice and immunofluorescence costaining of $\beta$-gal and A6 in liver of $A l b$-CreER $R^{T 2} ; R 26 R^{\text {lacz/+ }}$ mice after 3 weeks of DDC feeding, with percentages of marker-positive cells that coexpress YFP or $\beta$-gal. DNA was stained with DAPI (blue) (B and D). Data are expressed as means \pm SD. Scale bars: $100 \mu \mathrm{m}$ (A and C); $50 \mu \mathrm{m}$ (B and D). PV, portal vein.

analyses were performed using the data obtained by counting approximately 1000 cells per field of view in three discontinuous liver tissue slides for three individual mice.

\section{Flow Cytometry and PCR Analysis}

Single-cell suspensions of nonparenchymal cells were prepared from liver of TMX-administered CK19-CreER ${ }^{T 2}$; $R 26 R^{Y F P /+}$ mice after 2 weeks of DDC feeding and were incubated with phycoerythrin-conjugated anti-Ep-CAM monoclonal antibody (eBioscience) as described previously. ${ }^{12}$ The fluorescence-labeled cells were analyzed and separated with a FACSJazz cell sorter (BD Biosciences, San Jose, CA). PCR analyses were performed to determine whether Cremediated recombination occurred in the isolated cells, using their genomic DNA and the primers 5'-AAAGTCGCTCTGAGTTGTTAT-3' and 5'-TGGTAGCTCAGGTAGTGGTTGT- $3^{\prime}$ (forward and reverse, respectively).

\section{Results}

Hepatocytes, Rather than Cholangiocytes, Are the Major Source of Primitive Ductular Cells in a Mouse Model of Chronic Liver Injury

We crossed mouse lines expressing an inducible form of Cre recombinase $\left(\mathrm{CreER} \mathrm{R}^{T 2}\right)$ from the albumin genomic locus $\left(A l b-C r e E R^{T 2} \text { mice }\right)^{26}$ or the $C K 19$ genomic locus (CK19$\mathrm{CreER}^{T 2}$ mice), ${ }^{25}$ with $R 26 R^{\text {lacZlacZ }}$ or $R 26 R^{\text {YFP/YFP }}$ reporter mouse lines. ${ }^{27,28}$ In the double-mutant mice, administration of TMX allowed permanent marking of albumin-expressing or CK19-expressing cells and enabled us to follow the fate of the progeny of hepatocytes or cholangiocytes, respectively. ${ }^{25}$

After TMX injection, hepatocytes in liver of $\mathrm{Alb}-\mathrm{CreER} \mathrm{R}^{T 2}$; $R 26 R^{\text {lacZ/+ }}$ mice were labeled by lacZ, as assessed by X-gal staining to detect $\beta$-galactosidase ( $\beta$-gal) enzyme activity 

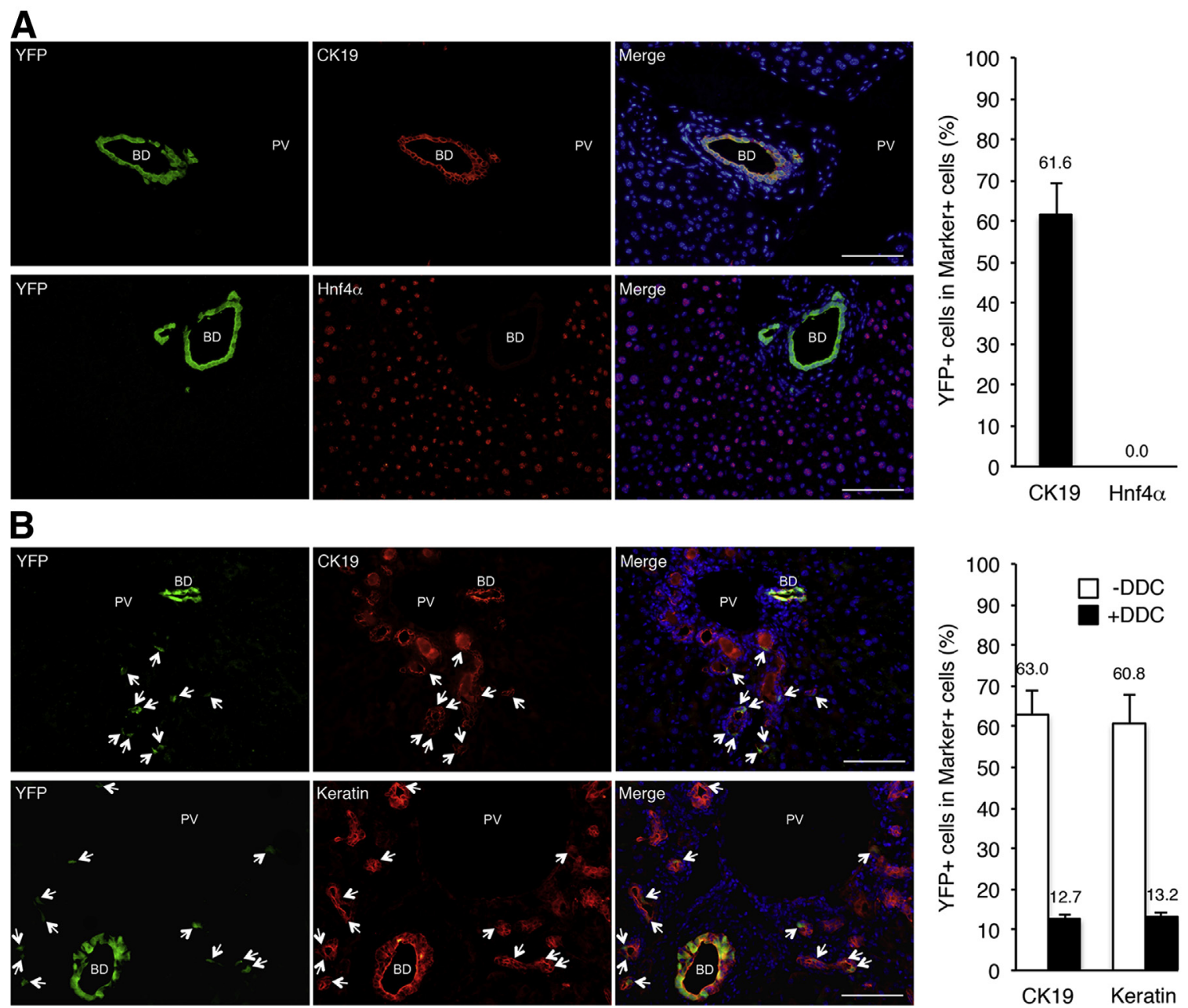

Figure 2 Only a small number of cells in DDC-induced primitive ductules are derived from cholangiocytes. A: Immunofluorescence staining of CK19 and Hnf4 $\alpha$ in liver of $C K 19-C r e E R^{T 2} ; R 26 R^{Y F P /+}$ mice fed a normal diet for 4 weeks after TMX injection, with percentages of CK19 ${ }^{+}$and Hnf $4 \alpha^{+}$cells that coexpress YFP. B: Immunofluorescence staining of CK19 and keratin in liver of $C K 19-C r e E R^{T 2} ; R 26 R^{Y F P /+}$ mice fed a normal diet for 1 week after TMX injection and then a DDC-containing diet for 3 weeks, with percentages of $\mathrm{CK}_{1} 9^{+}$and keratin ${ }^{+}$cells that coexpress YFP. YFP ${ }^{+}$cells (arrows) are evident in primitive ductules. DNA was stained with DAPI (blue). Data are expressed as means \pm SD. Scale bar $=100 \mu \mathrm{m}$. BD, bile duct; PV, portal vein.

(Figure 1A). By contrast, no lacZ $Z^{+}$cells were found within intrahepatic bile ducts or blood vessels, even at 4 weeks after TMX injection (Figure 1A). Moreover, in liver of Alb$C r e E R^{T 2} ; R 26 R^{Y F P /+}$ mice, YFP was expressed in almost all hepatocytes that were marked with the expression of hepatocyte nuclear factor $4 \alpha(\mathrm{Hnf} 4 \alpha)$, but not in any $\mathrm{CK} 19^{+}$cholangiocytes lining the intrahepatic bile ducts (Figure 1B). Thus, TMX injection led to specific labeling of hepatocytes and allowed us to trace the fate of lacZ $\mathrm{Z}^{+}$or $\mathrm{YFP}^{+}$hepatocyte progeny.

To induce chronic liver injury and primitive ductule formation in liver, we started feeding a $0.1 \%$ DDC-containing diet to $A l b-C r e E R^{T 2} ; R 26 R^{l a c Z /+}$ mice at 1 week after TMX injection. After 3 weeks of DDC feeding, many ductular structures appeared around the portal veins in liver of Alb-CreER ${ }^{T 2} ; R 26 R^{l a c Z /+}$ mice, and these primitive ductules consisted mainly of lac ${ }^{+}$cells (Figure $1 \mathrm{C}$ ). Moreover, the majority of DDC-induced primitive ductular cells observed in liver of $\mathrm{Alb}-\mathrm{CreER}{ }^{T 2} ; R 26 R^{Y F P /+}$ mice or $\mathrm{Alb}-\mathrm{CreER} \mathrm{R}^{T 2}$;
$R 26 R^{\text {lacZ/+ }}$ mice expressed YFP or $\beta$-gal with CK19, keratin, epithelial cell adhesion molecule (Ep-CAM), Trop2, and A6, which are antigens marking biliary lineage cells in normal and chronically injured livers ${ }^{29-31}$ (Figure 1D).

On the other hand, in liver of $C K 19-C r e E R^{T 2} ; R 26 R^{Y F P /+}$ mice, in which approximately $60 \%$ of cholangiocytes were specifically marked by YFP expression after TMX injection (Figure 2A), only a small number of cells within DDCinduced primitive ductules expressed YFP with CK19 and keratin (Figure 2B). To validate these findings, $\mathrm{YFP}^{+}$and $\mathrm{YFP}^{-}$cells among the Ep-CAM ${ }^{+}$biliary lineage cells were isolated from the nonparenchymal cell fraction in liver of TMX-administered $C K 19-\mathrm{CreER}^{T 2} ; R 26 R^{Y F P /+}$ mice after 2 weeks of DDC feeding. PCR analyses using genomic DNA from these cells revealed that Cre-mediated excision of the floxed termination sequence occurred in $\mathrm{YFP}^{+}$cells, but not in $\mathrm{YFP}^{-}$cells (Supplemental Figure S1).

Taken together, these findings demonstrate that both hepatocytes and cholangiocytes can be sources of the biliary 
A

\begin{tabular}{|c|c|}
\hline$+\mathrm{DDC}$ & $-\mathrm{DDC}$ \\
\hline 2 weeks & 15 weeks \\
\hline
\end{tabular}
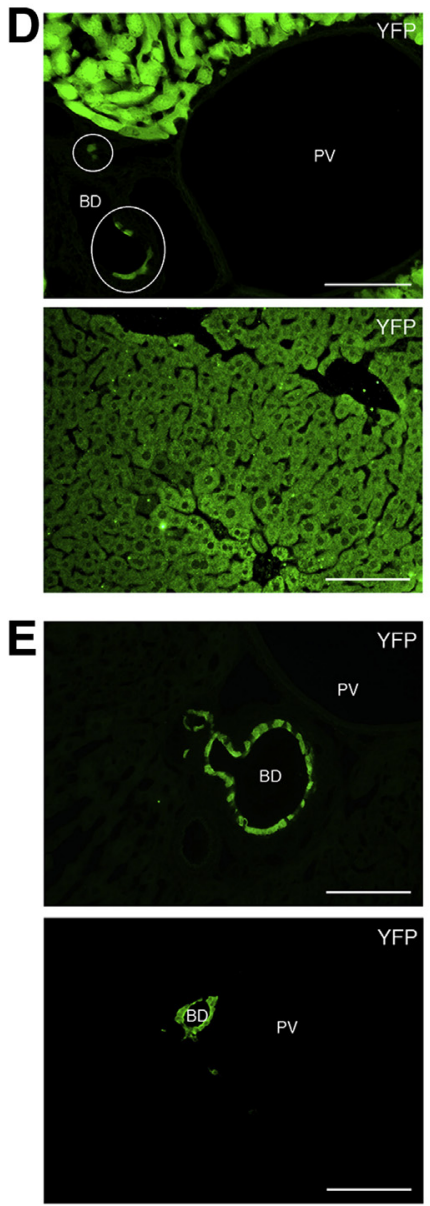
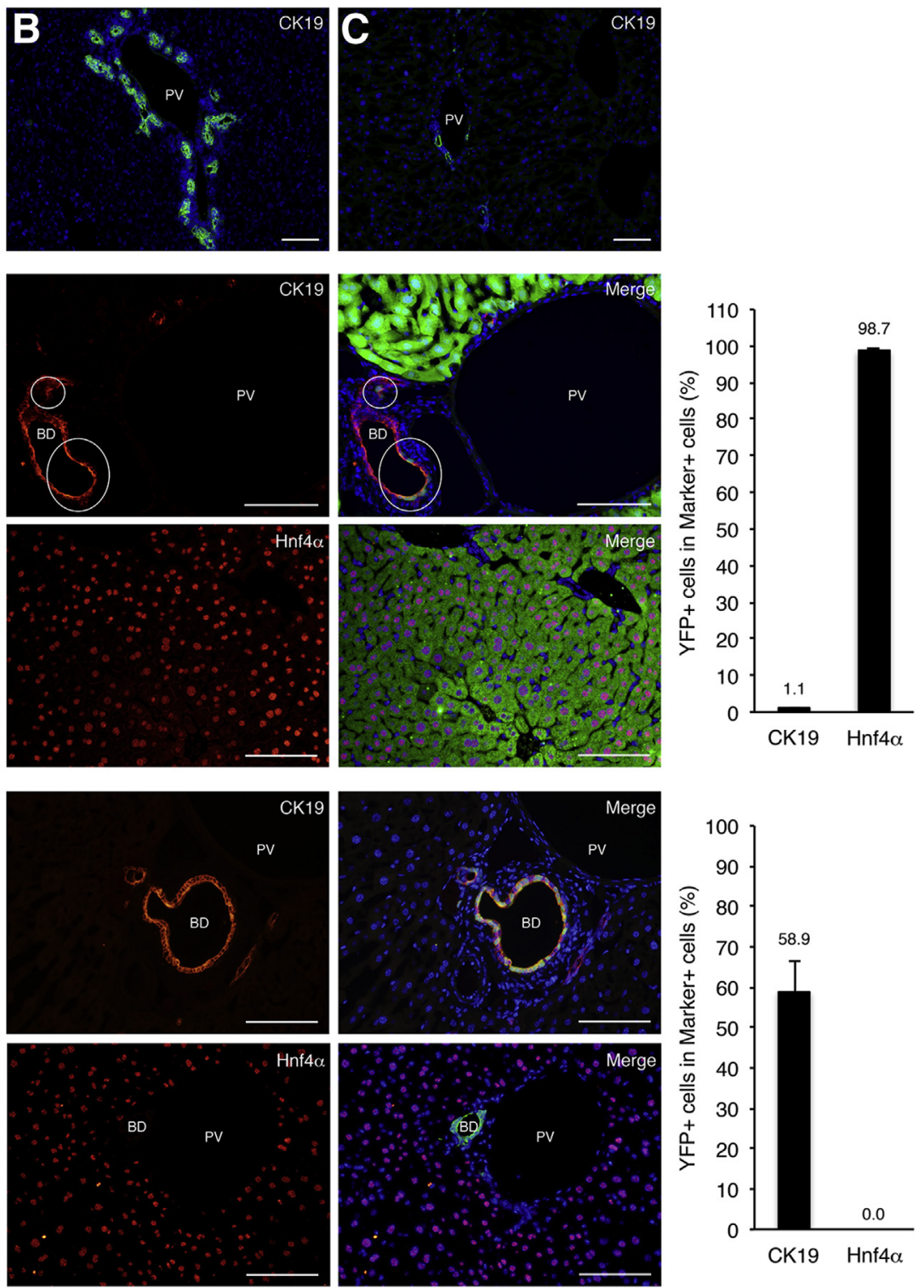

Figure 3 Hepatocyte-derived cells contribute to regeneration of intrahepatic bile ducts in recovery from chronic liver injury. A: Experimental procedure. To induce recovery from chronic injury induced by 2 weeks of DDC feeding, mice were fed a normal diet for 15 weeks. B and C: Immunofluorescence staining of CK19 in chronically injured liver of wild-type mice after 2 weeks of DDC feeding (B) or in injury-recovered liver after 2 weeks of DDC feeding followed by 15 weeks of a normal diet (C). In injury-recovered liver, no DDC-induced primitive ductules are observed, and only normal bile ducts (BD) remain around the portal veins (PV). DNA was stained with DAPI (blue). D and E: Immunofluorescence staining of CK19 and Hnf4 $\alpha$ in liver of Alb-CreER ${ }^{T 2} ; R 26 R^{Y F P /+}$ (D) and in liver of CK19$C r e E R^{T 2} ; R 26 R^{Y F P /+}$ mice $(\mathrm{E})$ after 2 weeks of DDC feeding followed by 15 weeks of a normal diet, with percentages of CK19 ${ }^{+}$and $\mathrm{Hnf} 4 \alpha^{+}$cells that coexpress YFP. Bile ducts containing YFP' hepatocyte-derived cells are circled. DNA was stained with DAPI (blue). Data are expressed as means \pm SD. Scale bar $=100 \mu \mathrm{m}$.

lineage cells that form primitive ductules in a mouse model of chronic liver injury, although the majority of these cells arise from hepatocytes, rather than cholangiocytes.

\section{A Slight Contribution of Hepatocyte-Derived Cells to} Bile Duct Regeneration in Liver Tissue Recovered from Chronic Injury

We next investigated what occurs in liver tissues after cessation of DDC treatment. In recovery from chronic injury, DDC-induced primitive ductules disappeared from the liver during subsequent feeding of a normal diet for 15 weeks, and only intrahepatic bile ducts remained around the portal veins (Figure 3, A-C). Lineage-tracing analyses using $A l b$ $\operatorname{CreER}^{T 2} ; R 26 R^{Y F P /+}$ mice revealed that $\mathrm{YFP}$ was still expressed in almost all $\mathrm{Hnf} 4 \alpha^{+}$hepatocytes, and that $\mathrm{YFP}^{+}$ hepatocyte-derived cells could also contribute (but rarely) to the formation of bile ducts as $\mathrm{CK} 19^{+}$cholangiocytes, in liver tissues after recovery from DDC-induced chronic injury (Figure 3D). On the other hand, in liver tissues of CK19$\mathrm{CreER}^{T 2} ; R 26 \mathrm{R}^{Y F P /+}$ mice after recovery from chronic injury, approximately $60 \%$ of cholangiocytes still expressed YFP, but any $\mathrm{Hnf} 4 \alpha^{+}$hepatocytes did not express YFP (Figure $3 \mathrm{E}$ ). These findings demonstrate that, in recovery from 

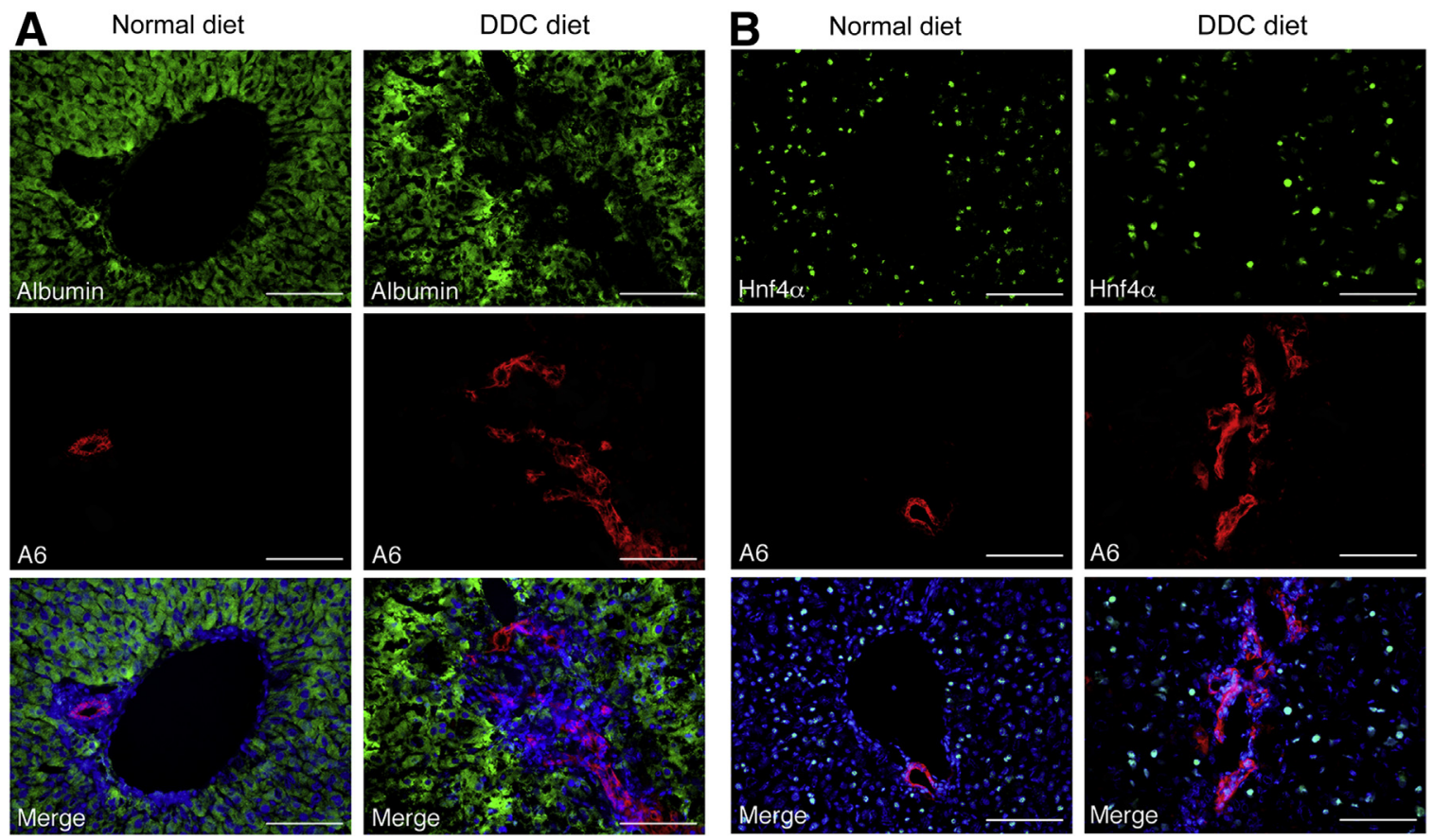

C
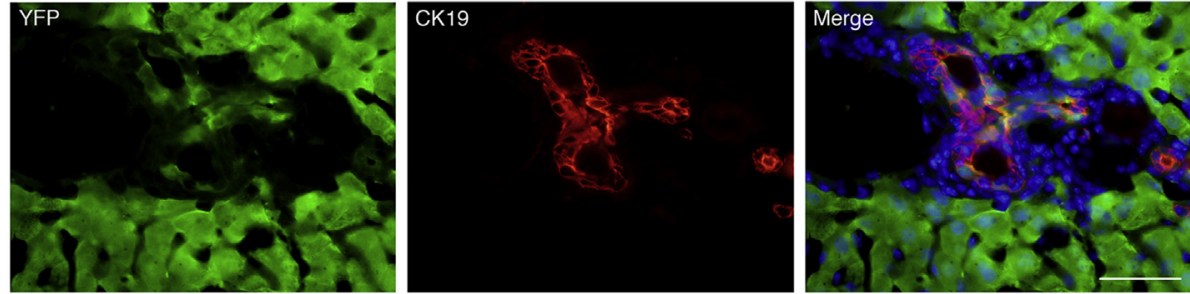

Figure 4 Neither albumin nor $\mathrm{Hnf} 4 \alpha$ is expressed in primitive ductular cells even during the early stage of chronic liver injury, although these ductular cells are derived mainly from hepatocytes. A and B: Immunofluorescence costaining of A6 and albumin (A) or Hnf $4 \alpha$ (B) in liver of wild-type mice fed a normal diet or a DDC-containing diet for 1 week. C: Immunofluorescence staining of CK19 in liver of $A l b$-CreER ${ }^{T 2} ; R 26 R^{Y F P /+}$ mice after 1 week of DDC feeding. DNA was stained with DAPI (blue). Scale bars: $100 \mu \mathrm{m}$ (A and B); $50 \mu \mathrm{m}$ (C).

DDC-induced chronic liver injury, hepatocytes arise from hepatocyte-derived cells, but not from cholangiocyte-derived cells, and bile duct regeneration occurs by self-duplication of cholangiocytes, with only a slight contribution from hepatocytederived cells.

\section{Hepatocytes Actually Contribute to Primitive Ductule Formation in Chronically Injured Liver}

In the rat 2-acetylaminofluorene liver injury model, biliary lineage cells within the primitive ductules are, at least in part, positive for albumin expression. ${ }^{32}$ Thus, in the mouse DDC model, newly generated ductular cells might also express albumin in an early phase of ductular structure formation and be labeled by the remnant TMX. However, this possibility can be excluded, for several reasons. First, the efficacy of TMX is rapid and short lived, disappearing within 2 days, ${ }^{33,34}$ and therefore TMX is no longer effective at the beginning of DDC feeding in this model and just induces labeling of hepatocytes in the normal liver. Indeed, accumulation of Cre recombinase in the nuclei of hepatocytes was not observed in liver of $A l b-C r e E R^{T 2}$ mice at 1 week after TMX injection, when we normally started feeding the DDC-containing diet to mice (Supplemental Figure S2). Moreover, even though we started DDC feeding at 2 weeks after TMX injection, primitive ductular cells in liver of $A l b$ CreER ${ }^{T 2} ; R 26 R^{Y F P /+}$ mice were $\mathrm{YFP}^{+}$(Supplemental Figure S3). Second, the $\mathrm{A}^{+}$ductular cells that appeared after 1 week of DDC feeding did not express either albumin or $\mathrm{Hnf} 4 \alpha$, although most of these ductular cells were derived from hepatocytes (Figure 4). Third, all of the primitive ductular cells in DDC-treated mouse liver expressed CD133 (prominin-1) (a five-transmembrane cell surface glycoprotein $^{12}$ ). Our research group has previously isolated these $\mathrm{CD} 133^{+}$ductular cells by flow cytometry and examined the expression of markers for hepatocytes and biliary lineage cells; the results showed that freshly isolated $\mathrm{CD} 133^{+}$cells expressed CK19 and CK7, but did not express any hepatocyte markers, including albumin. ${ }^{12}$ There is also a possibility that TMX-independent Cre activation can occur in DDCtreated mouse liver, in which case the primitive ductules formed in chronically injured liver would arise from other than hepatocytes. To address this possibility, we fed the DDC-containing diet to Alb-CreER ${ }^{T 2} ; R 26 R^{Y F P /+}$ mice 

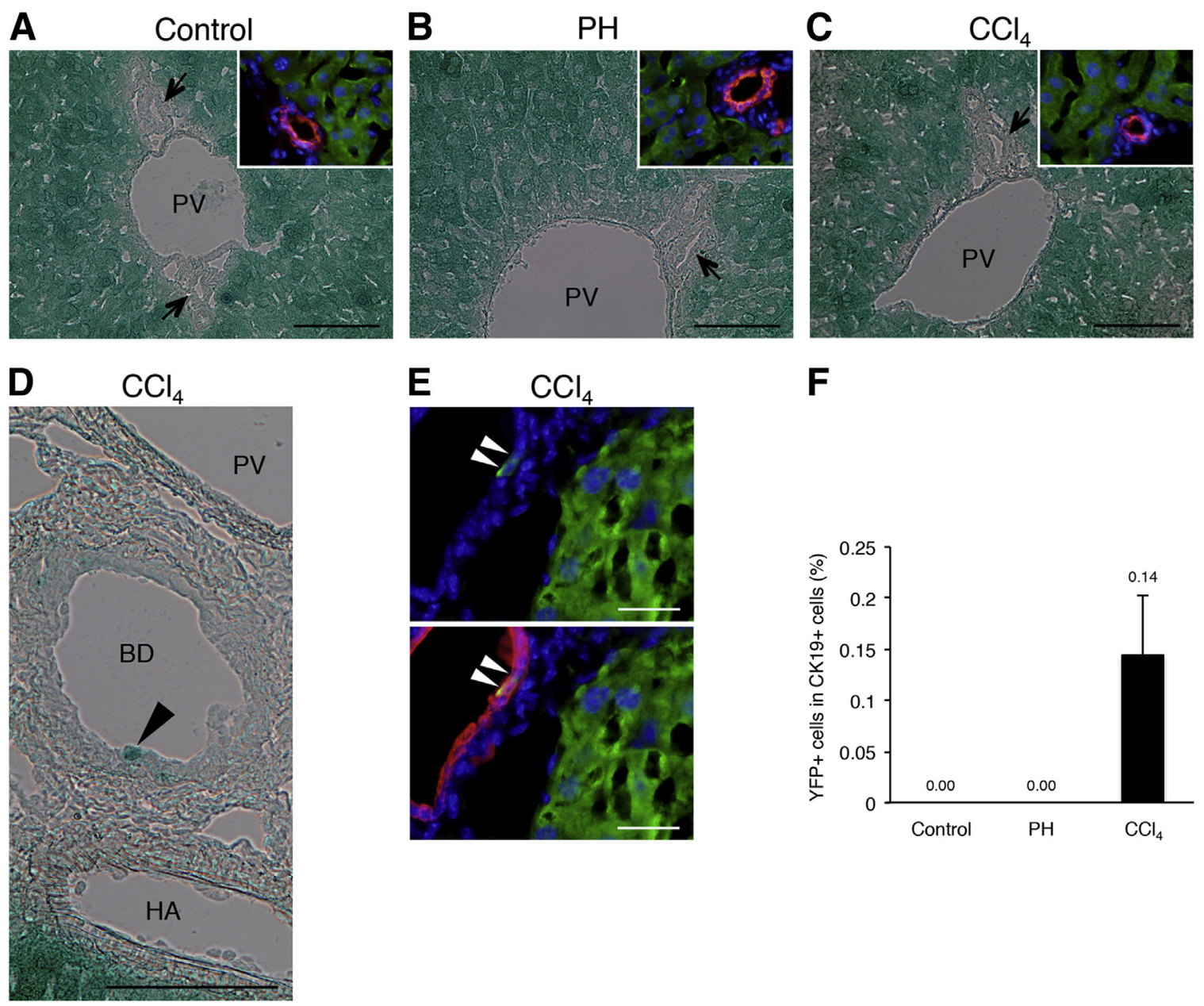

$\mathbf{F}$

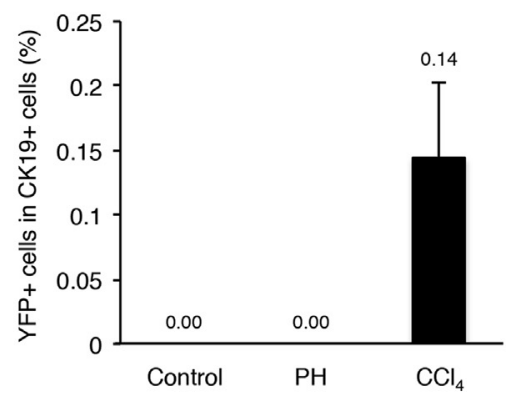

Figure 5 Hepatocytes are rarely converted into biliary lineage cells within intrahepatic bile ducts after fulminant hepatic failure, and are not converted after PH. A-E: X-gal staining (A-D) and immunofluorescence staining of CK19 (red signal in insets of panels A-C and in the bottom panel of E) in liver of $A l b-C r e E R^{T 2} ; R 26 R^{l a c Z /+}$ mice $(\mathbf{A}-\mathbf{C}$, main images, and $\mathbf{D})$ and $A l b-C r e E R^{T 2} ; R 26 R^{Y F P /+}$ mice $(\mathbf{A}-\mathbf{C}$, insets, and $\mathbf{E})$ at 3 weeks after TMX injection $(\mathbf{A})$ and at 2 weeks after $\mathrm{PH}(\mathbf{B})$ or $\mathrm{CCl}_{4}$ administration (C-E) performed 1 week after TMX injection. The majority of bile ducts do not contain any lacZ ${ }^{+}$or $\mathrm{YFP}^{+}$cells after $\mathrm{CCl}_{4}$-induced fulminant hepatic failure (C). Intrahepatic bile ducts composed of lacZ $Z^{-}$cells (arrows) are evident $(\mathbf{A}-\mathbf{C})$. A small number of bile ducts contain a few lacZ ${ }^{+}$or YFP ${ }^{+}$cells (arrowheads) (D and E). F: The proportion of $\mathrm{CK}_{1} 9^{+}$cells that coexpress YFP in control mouse liver and in liver regenerated after PH or $\mathrm{CCl}_{4}$ administration. DNA was stained with DAPI (blue). Scale bars: $100 \mu \mathrm{m}(\mathbf{A}-\mathbf{D}) ; 25 \mu \mathrm{m}(\mathbf{E})$. BD, bile duct; HA, hepatic artery; PV, portal vein.

without TMX injection. After 3 weeks of DDC feeding, we did not find any $\mathrm{YFP}^{+}$cells in liver of these mice, which suggests that DDC did not induce TMX-independent Cre activity in the liver (Supplemental Figure S4). Taken together, these findings demonstrate that hepatocytes contribute to primitive ductule formation in DDC-treated chronically injured mouse liver.

\section{Hepatocytes Are Rarely Converted into Biliary Lineage} Cells within Intrahepatic Bile Ducts after Fulminant Hepatic Failure

Given these findings, we further investigated whether hepatocytes contribute to biliary ductule reconstitution during normal liver regeneration after partial hepatectomy $(\mathrm{PH})$ or fulminant hepatic failure caused by administration of hepatotoxic chemicals. At 1 week after TMX injection into $A l b$ -
$\mathrm{CreER}^{T 2} ; R 26 R^{\text {lacZ/+ }}$ mice and Alb-CreER ${ }^{T 2} ; R 26 R^{Y F P /+}$ mice, we performed PH (removal of $70 \%$ of the liver lobe) or subcutaneously injected carbon tetrachloride $\left(\mathrm{CCl}_{4}\right)$ dissolved in olive oil ( $2 \mathrm{~mL} / \mathrm{kg}$ mouse weight). As expected, no ductular structures other than normal bile ducts were observed in regenerated liver tissues analyzed at 2 weeks after $\mathrm{PH}$ or chemical injury. After PH treatment, lacZ and YFP were detected only in hepatocytes, similar to the normal liver (Figure 5, A and B). However, in liver tissues regenerated after fulminant hepatic failure, lacZ $Z^{+}$or $\mathrm{YFP}^{+}$cells resided within bile ducts, although the frequencies of these lac $\mathrm{Z}^{+}$or $\mathrm{YFP}^{+}$ cholangiocytes were extremely low (Figure 5, C-F). These findings demonstrate that, in addition to DDC-induced chronic injury, $\mathrm{CCl}_{4}$-induced fulminant hepatic failure can be a trigger for the generation of hepatocyte-derived biliary lineage cells (although this effect may be too weak to induce primitive ductule formation from hepatocytes). 

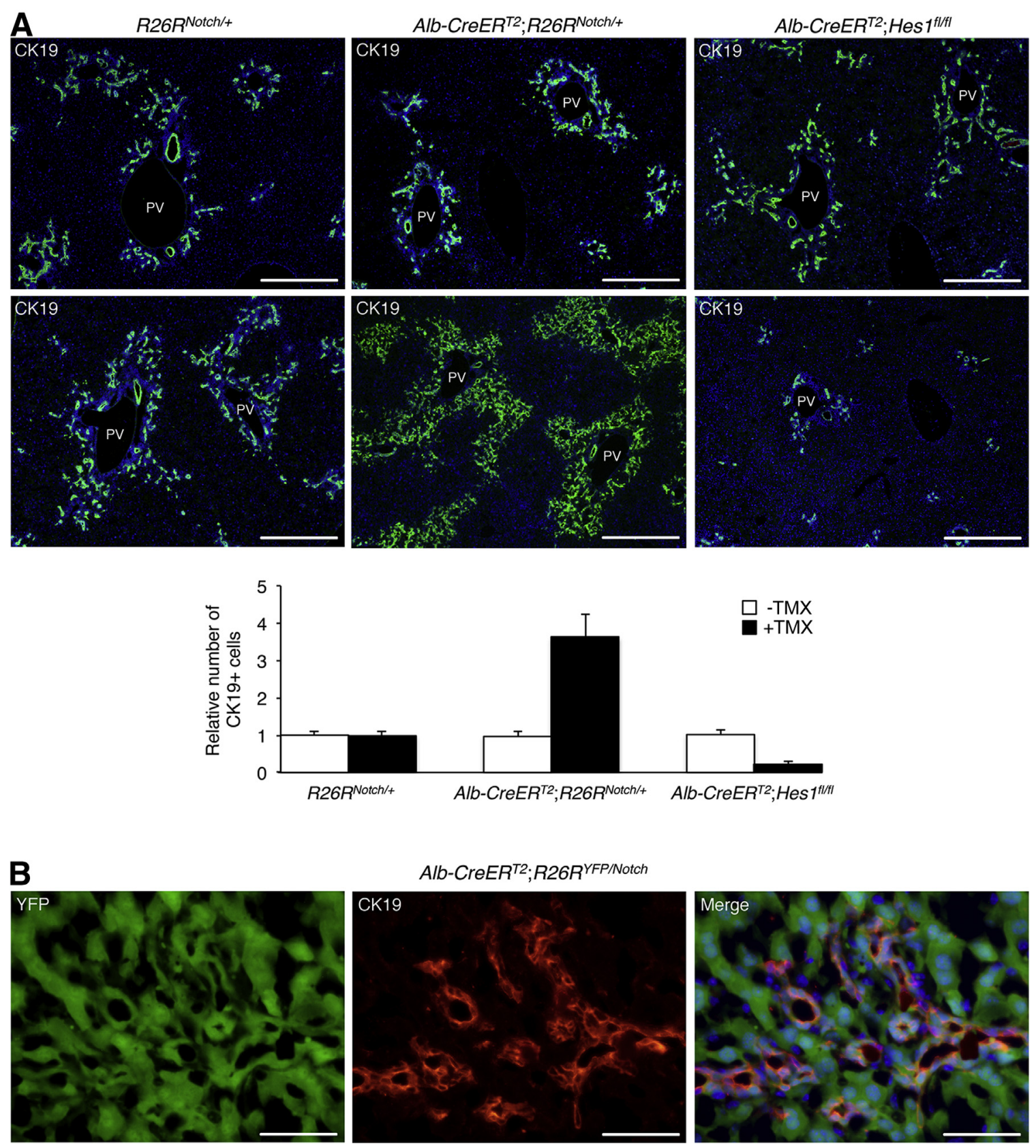

Figure 6 Activation of the Notch-Hes1 signaling axis is important for conversion of hepatocytes into primitive ductular cells in chronically injured liver. A: Immunofluorescence staining of CK19 in liver of $R 26 R^{\text {Notch } /+}, A l b-C r e E R^{T 2} ; R 26 R^{N o t c h /+}$, and $A l b-C r e E R^{T 2}$; Hes $1^{f l f l}$ mice after 3 weeks of DDC feeding without (top row) or with (bottom row) prior TMX injection. DNA was stained with DAPI (blue). To show fold differences (relative number) of CK19+ cells per portal vein, data were normalized by the value of the liver of $R 26 R^{N o t c h /+}$ mice after 3 weeks of DDC feeding without prior TMX injection. B: Immunofluorescence staining of CK19 in liver of $A l b-C r e E R^{T 2} ; R 26 R^{Y F P / N o t c h}$ mice after 2 weeks of DDC feeding. Data are expressed as means \pm SD. Scale bars: $500 \mu \mathrm{m}(\mathbf{A}) ; 100 \mu \mathrm{m}(\mathbf{B})$. PV, portal vein.

Activation of the Notch-Hes1 Signaling Axis Is Important for Conversion of Hepatocytes into Primitive Ductular Cells in Chronically Injured Liver

Next, we focused on the mechanism controlling the conversion of hepatocytes into primitive ductular cells in DDCinduced chronic liver injury. Activation of Notch signaling is important for induction of the biliary program, not only in hepatic progenitor cells during development, but also in postnatal hepatocytes..$^{21,25,35,36}$ In some of these studies, both DDC-induced chronic liver injury and enforced Notch signal activation induced expression of the transcription factor SOX-9 (Sox9) in hepatocytes and subsequently changed their morphology to that of biliary lineage cells. $^{21,36}$ In the present study, we also observed Sox9 expression in $\mathrm{Hnf} 4 \alpha^{+}$periportal hepatocytes after 5 days of DDC feeding and in hepatocytes adjacent to primitive ductular cells after 1 week of DDC feeding (Supplemental Figure S5, A and B). Moreover, lineage-tracing analyses using $\mathrm{Alb}-\mathrm{CreER}{ }^{T 2} ; R 26 R^{Y F P /+}$ mice showed that Sox9 was 
expressed in $\mathrm{YFP}^{+}$hepatocyte-derived cells after 1 week of DDC feeding (Supplemental Figure S5C). However, we could not observe primitive ductules in liver of TMXadministered $\mathrm{Alb}-\mathrm{CreER}^{T 2} ; R 26 R^{\mathrm{Notch} /+}$ mice, in which hepatocytes hemizygotically express an intracellular fragment of mouse Notch1 (NotchIC) ${ }^{37}$ with constitutive Notch signaling activity, at 5 weeks after TMX injection (Supplemental Figure S6A). Given that primitive ductules appeared in liver of these mice at 15 weeks after TMX injection, a weak expression of NotchIC is not sufficient to induce a rapid conversion of hepatocytes into primitive ductular cells (Supplemental Figure S6B). We therefore sought to determine whether such a weak activation of Notch signaling is synergistically effective for conversion of hepatocytes into primitive ductular cells during DDCinduced chronic liver damage. It was recently reported that the transcription factor RBP-J $\kappa$, which is activated by the binding of NotchIC, is important for hepatocyte conversion to the DDC-induced primitive ductular cells. ${ }^{21}$ However, because RBP-JK regulates the expression of various genes, including the genes encoding the Hes family of transcription factors, the specific target gene of RBP-JK in hepatocytes remained to be identified. Previously, our research group reported that Hesl is essential for the conversion of hepatocytes into biliary lineage cells at the onset of intrahepatic cholangiocarcinoma. ${ }^{25}$ Thus, in the present study, we investigated whether Hesl (the target gene of RBP-JK) is also important for the conversion of hepatocytes into primitive ductular cells in DDC-treated chronically injured liver.

To address these issues, we induced primitive ductule formation in liver of TMX-administered $\mathrm{Alb}-\mathrm{CreER}^{T 2}$; $R 26 R^{\text {Notch/+ }}$ mice and Alb-CreER ${ }^{T 2} ; H_{e s} I^{f / f l}$ mice, in which the hepatocytes lack the gene encoding Hes $1,{ }^{38}$ by feeding the DDC-containing diet for 3 weeks. Immunofluorescence analyses revealed that the number of $\mathrm{CK} 19^{+}$cells forming primitive ductules around portal veins was significantly increased in $A l b-C r e E R^{T 2} ; R 26 R^{\text {Notch } /+}$ mice and was significantly decreased in $A l b-C_{C H E R}{ }^{T 2} ; H e s I^{f l f l}$ mice (Figure 6A). Moreover, lineage-tracing analyses using $A l b$ $\mathrm{CreER}^{T 2} ; R 26 R^{Y F P / N o t c h}$ mice confirmed that the $\mathrm{CK} 19^{+}$ cells increased due to Notch signal activation were derived from hepatocytes (Figure 6B). Taken together, these findings demonstrate that a weak activation of Notch signaling in hepatocytes synergistically enhances the DDC-induced hepatocyte conversion to primitive ductular cells and that activation of the Notch-Hes1 signaling axis is important for such conversion in the mouse DDC model of chronic liver injury.

\section{Discussion}

The present results demonstrate that both albumin-positive hepatocytes and $\mathrm{CK} 19^{+}$cholangiocytes in liver can be sources of the biliary lineage cells that form primitive ductules in the DDC mouse model of chronic liver injury. However, the majority of these biliary lineage cells were derived from hepatocytes, rather than from cholangiocytes. This observation is consistent with previous findings that only a proportion of the cells in the primitive ductules are derived from $\operatorname{Sox}^{+}{ }^{+}$or osteopontin ${ }^{+}$cholangiocytes in DDCtreated chronically injured liver. ${ }^{15-17}$ In the present study, most of the residual cells within DDC-induced primitive ductules originated from hepatocytes. Although Yanger et $\mathrm{al}^{21}$ also reported that hepatocytes could be converted into primitive ductular cells in response to chronic liver damage, the percentage of hepatocyte-derived primitive ductular cells in their study was much lower than in our present study. Moreover, our results showing conversion of hepatocytes into primitive ductular cells are disparate from reports that transplanted mouse hepatocytes in the chimeric liver and the population of $\mathrm{Ttr}^{+}$hepatocytes in the normal mouse liver did not give rise to primitive ductular cells in the DDC model. ${ }^{22,23}$ Possible reasons for these critical differences include that i) AAV-mediated gene transfer to the liver (used in the studies of Yanger et $\mathrm{al}^{21}$ and Malato et $\mathrm{al}^{23}$ ) may suppress the conversion of hepatocytes into primitive ductular cells in DDC-induced chronic liver injury; ii) the recipient mutant hepatocytes in the chimeric liver may be predominantly converted into the primitive ductular cells after DDC treatment, rather than transplanted normal hepatocytes; and iii), given that a region of the $T B G$ promoter (Yanger et $\mathrm{al}^{21}$ ) or the Ttr promoter (Malato et $\mathrm{al}^{23}$ ) was used to drive Cre expression, not all TBG/Ttr ${ }^{+}$cells may exhibit Cre activity, and albumin-positive but TBG/Ttr ( $\mathrm{Cre}$ )-negative hepatocytes may be identified in liver.

On the other hand, it is also possible that hepatocytes undergo a qualitative alteration after TMX injection and/or chronic $\mathrm{CreER}^{T 2}$ expression in our experimental system. Specifically, such an abnormal condition could induce conversion of intact hepatocytes into putative lineageconvertible hepatocytes, which could then change into primitive ductular cells after DDC feeding. However, the numbers of primitive ductular cells induced in the mouse DDC model were almost the same among mice with or without TMX injection and chronic CreER ${ }^{T 2}$ expression. Moreover, Yanger et $\mathrm{al}^{21}$ showed that hepatocytes could be converted into biliary lineage cells in the mouse DDC model without TMX injection and chronic $C r e E R^{T 2}$ expression. Thus, it is difficult to conclude that lineage-convertible hepatocytes appear in response to TMX injection and/or chronic $\mathrm{CreER}^{T 2}$ expression for the conversion of hepatocytes to primitive ductular cells after DDC feeding.

In the present study, we found a synergistic effect of Notch signal activation in DDC-induced chronic liver injury, which was important for the induction of primitive ductule formation from albumin-positive hepatocytes. In liver of TMX-administered Alb-CreER ${ }^{T 2} ; R 26 R^{\text {Notch/+ }}$ mice, Notch signal activation in hepatocytes may confer the potential to change the fate to biliary lineage cells on all hepatocytes. However, it may still be insufficient for induction of the 
efficient conversion of hepatocytes into $\mathrm{CK} 19^{+}$morphologically identified biliary lineage cells. Thus, additional stimuli caused by DDC-induced chronic liver injury, including an additional Notch signal activation, may increase the number of primitive ductular cells in combination with a basal activation of Notch signaling in $\mathrm{Alb}-\mathrm{CreER}^{T 2} ; R 26 R^{\mathrm{Notch} /+}$ mouse hepatocytes. In $\mathrm{Alb}-\mathrm{CreER^{T2 }} ; R 26 R^{\text {Notch/+ }}$ mice, even though Notch signaling was activated in all hepatocytes, only those hepatocytes residing around the portal areas were able to convert into biliary lineage cells and form primitive ductules. This finding suggests that there may be a unique population of hepatocytes in the portal areas of the hepatic lobule. Indeed, it was reported that hepatocytes around the portal veins can convert into biliary lineage cells when intrahepatic bile duct regeneration is induced, whereas cholangiocytes cannot proliferate owing to toxic influences. ${ }^{19}$ In a previous study, however, our research group demonstrated that hepatocytes residing around the central veins can also convert into biliary lineage cells after administration of thioacetamide. ${ }^{25}$ Thus, hepatocytes may be capable of changing their fate to biliary lineage cells regardless of their position in the hepatic lobule; however, the particular location of lineage-converting hepatocytes should be decided by the nature of the toxins used.

We also identified Hes1 as a critical effector of Notch signaling in hepatocyte conversion to DDC-induced primitive ductular cells. The Hes 1 gene is regulated by RBP-J $\kappa$, which is activated by the binding of NotchIC and important for conversion of hepatocytes into primitive ductular cells during DDC-induced chronic liver damage. ${ }^{21}$ Thus, activation of the Notch-RBP-JK-Hes1 signaling axis in hepatocytes should be crucial for conversion of hepatocytes into DDC-induced primitive ductular cells. Moreover, because the number of primitive ductular cells induced after DDC feeding was decreased in $\mathrm{Alb}-\mathrm{CreER}^{T 2} ; \mathrm{Hes}^{\mathrm{flfl}}$ mice, hepatocytes, but not other types of cells in liver, could be considered as the major source of the biliary lineage cells that form primitive ductules in the mouse DDC model of chronic liver injury.

In conclusion, the present results indicate that albuminpositive hepatocytes residing in the portal areas, rather than CK19 ${ }^{+}$cholangiocytes lining the intrahepatic bile ducts, give rise to the majority of the biliary lineage cells that form primitive ductules in DDC-induced chronically injured liver, through Notch-mediated cell lineage conversion. Interestingly, in recovery from chronic liver injury, hepatocytederived cells could be involved in bile duct regeneration as $\mathrm{CK} 19^{+}$cholangiocytes; however, the frequency was low. Moreover, the present results show that, to a lesser extent, hepatocytes could also be converted into cholangiocytes through integration into intrahepatic bile ducts after fulminant hepatic failure. By contrast, we could not find any cholangiocyte-derived hepatocytes in liver tissues recovered from DDC-induced chronic liver injury, which is consistent with results from a previous study. ${ }^{17}$ However, it has also been shown that cholangiocyte-derived cells can be a source of hepatocytes in recovery from CDE-induced chronic liver injury. ${ }^{17}$ Thus, the potential of primitive ductular cells in chronically injured liver may differ across experimental models of chronic liver injury. Alternatively, because the number of cholangiocyte-derived hepatocytes increased when the amount of the extracellular matrix in liver was reduced during CDE-induced chronic liver damage, ${ }^{17}$ it may be that the difference between the DDC and CDE models of chronic liver injury is based only on differences in the quality and quantity of extracellular matrix in these models. Elucidation of the mechanisms underlying the fate conversion of hepatocytes in liver diseases is needed for understanding why and how hepatocytes change their fate to primitive ductular cells. Such understanding could facilitate development of therapeutic strategies for liver diseases.

\section{Acknowledgments}

We thank Drs. Pierre Chambon, Daniel Metzger, Frank Costantini, Douglas A. Melton, Ryoichiro Kageyama, and Valentina M. Factor for sharing reagents and providing mice and Masuyo Kawabata, Momoko Hamano, Chiaki Kaieda, and Masato Tanaka for technical assistance.

\section{Supplemental Data}

Supplemental material for this article can be found at http://dx.doi.org/10.1016/j.ajpath.2014.01.005.

\section{References}

1. Lowes KN, Brennan BA, Yeoh GC, Olynyk JK: Oval cell numbers in human chronic liver diseases are directly related to disease severity Am J Pathol 1999, 154:537-541

2. Prior P: Long-term cancer risk in alcoholism. Alcohol Alcohol 1988, $23: 163-171$

3. Tsukuma H, Hiyama T, Tanaka S, Nakao M, Yabuuchi T, Kitamura T, Nakanishi K, Fujimoto I, Inoue A, Yamazaki H, Kawashima T: Risk factors for hepatocellular carcinoma among patients with chronic liver disease. N Engl J Med 1993, 328:1797-1801

4. Deugnier YM, Guyader D, Crantock L, Lopez JM, Turlin B, Yaouanq J, Jouanolle H, Campion JP, Launois B, Halliday JW, Powell LW, Brissot P: Primary liver cancer in genetic hemochromatosis: a clinical, pathological, and pathogenetic study of 54 cases. Gastroenterology 1993, 104:228-234

5. Farber E: Similarities in the sequence of early histological changes induced in the liver of the rat by ethionine, 2-acetylamino-fluorene, and 3'-methyl-4-dimethylaminoazobenzene. Cancer Res 1956, 16:142-148

6. Shinozuka H, Lombardi B, Sell S, Iammarino RM: Early histological and functional alterations of ethionine liver carcinogenesis in rats fed a choline-deficient diet. Cancer Res 1978, 38:1092-1098

7. Lemire JM, Shiojiri N, Fausto N: Oval cell proliferation and the origin of small hepatocytes in liver injury induced by D-galactosamine. Am J Pathol 1991, 139:535-552

8. Tatematsu M, Ho RH, Kaku T, Ekem JK, Farber E: Studies on the proliferation and fate of oval cells in the liver of rats treated with 2acetylaminofluorene and partial hepatectomy. Am J Pathol 1984, 114:418-430

9. Preisegger KH, Factor VM, Fuchsbichler A, Stumptner C, Denk H, Thorgeirsson SS: Atypical ductular proliferation and its inhibition by transforming growth factor beta1 in the 3,5-diethoxycarbonyl-1,4- 
dihydrocollidine mouse model for chronic alcoholic liver disease. Lab Invest 1999, 79:103-109

10. Factor VM, Radaeva SA: Oval cells-hepatocytes relationships in Dipin-induced hepatocarcinogenesis in mice. Exp Toxicol Pathol 1993, 45:239-244

11. Dumble ML, Croager EJ, Yeoh GC, Quail EA: Generation and characterization of $\mathrm{p} 53$ null transformed hepatic progenitor cells: oval cells give rise to hepatocellular carcinoma. Carcinogenesis 2002, 23:435-445

12. Suzuki A, Sekiya S, Onishi M, Oshima N, Kiyonari H, Nakauchi H, Taniguchi H: Flow cytometric isolation and clonal identification of self-renewing bipotent hepatic progenitor cells in adult mouse liver. Hepatology 2008, 48:1964-1978

13. Fausto N, Campbell JS: The role of hepatocytes and oval cells in liver regeneration and repopulation. Mech Dev 2003, 120:117-130

14. Michalopoulos GK: Liver regeneration: alternative epithelial pathways. Int J Biochem Cell Biol 2011, 43:173-179

15. Furuyama K, Kawaguchi Y, Akiyama H, Horiguchi M, Kodama S, Kuhara T, Hosokawa S, Elbahrawy A, Soeda T, Koizumi M, Masui T, Kawaguchi M, Takaori K, Doi R, Nishi E, Kakinoki R, Deng JM, Behringer RR, Nakamura T, Uemoto S: Continuous cell supply from a Sox9-expressing progenitor zone in adult liver, exocrine pancreas and intestine. Nat Genet 2011, 43:34-41

16. Dorrell C, Erker L, Schug J, Kopp JL, Canaday PS, Fox AJ, Smirnova O, Duncan AW, Finegold MJ, Sander M, Kaestner KH, Grompe M: Prospective isolation of a bipotential clonogenic liver progenitor cell in adult mice. Genes Dev 2011, 25:1193-1203

17. Español-Suñer R, Carpentier R, Van Hul N, Legry V, Achouri Y, Cordi S, Jacquemin P, Lemaigre F, Leclercq IA: Liver progenitor cells yield functional hepatocytes in response to chronic liver injury in mice. Gastroenterology 2012, 143:1564-1575

18. Michalopoulos GK, Bowen WC, Mulè K, Lopez-Talavera JC, Mars W: Hepatocytes undergo phenotypic transformation to biliary epithelium in organoid cultures. Hepatology 2002, 36:278-283

19. Michalopoulos GK, Barua L, Bowen WC: Transdifferentiation of rat hepatocytes into biliary cells after bile duct ligation and toxic biliary injury. Hepatology 2005, 41:535-544

20. Nishikawa Y, Doi Y, Watanabe H, Tokairin T, Omori Y, Su M, Yoshioka T, Enomoto K: Transdifferentiation of mature rat hepatocytes into bile duct-like cells in vitro. Am J Pathol 2005, 166:1077-1088

21. Yanger K, Zong Y, Maggs LR, Shapira SN, Maddipati R, Aiello NM, Thung SN, Wells RG, Greenbaum LE, Stanger BZ: Robust cellular reprogramming occurs spontaneously during liver regeneration [Erratum appeared in Genes Dev 2013, 27:1537]. Genes Dev 2013, 27 : $719-724$

22. Wang $\mathrm{X}$, Foster $\mathrm{M}$, Al-Dhalimy $\mathrm{M}$, Lagasse E, Finegold $\mathrm{M}$, Grompe M: The origin and liver repopulating capacity of murine oval cells. Proc Natl Acad Sci USA 2003, 100(Suppl 1):11881-11888

23. Malato Y, Naqvi S, Schürmann N, Ng R, Wang B, Zape J, Kay MA, Grimm D, Willenbring H: Fate tracing of mature hepatocytes in mouse liver homeostasis and regeneration. J Clin Invest 2011, 121: $4850-4860$

24. Petersen BE, Bowen WC, Patrene KD, Mars WM, Sullivan AK, Murase N, Boggs SS, Greenberger JS, Goff JP: Bone marrow as a potential source of hepatic oval cells. Science 1999, 284:1168-1170

25. Sekiya S, Suzuki A: Intrahepatic cholangiocarcinoma can arise from Notch-mediated conversion of hepatocytes. J Clin Invest 2012, 122 : 3914-3918

26. Schuler M, Dierich A, Chambon P, Metzger D: Efficient temporally controlled targeted somatic mutagenesis in hepatocytes of the mouse Genesis 2004, 39:167-172

27. Soriano P: Generalized lacZ expression with the ROSA26 Cre reporter strain. Nat Genet 1999, 21:70-71

28. Srinivas S, Watanabe T, Lin CS, William CM, Tanabe Y, Jessell TM, Costantini F: Cre reporter strains produced by targeted insertion of EYFP and ECFP into the ROSA26 locus. BMC Dev Biol 2001, 1:4

29. de Boer CJ, van Krieken JH, Janssen-van Rhijn CM, Litvinov SV: Expression of Ep-CAM in normal, regenerating, metaplastic, and neoplastic liver. J Pathol 1999, 188:201-206

30. Okabe M, Tsukahara Y, Tanaka M, Suzuki K, Saito S, Kamiya Y, Tsujimura T, Nakamura K, Miyajima A: Potential hepatic stem cells reside in EpCAM+ cells of normal and injured mouse liver. Development 2009, 136:1951-1960

31. Engelhardt NV, Factor VM, Yasova AK, Poltoranina VS, Baranov VN, Lasareva MN: Common antigens of mouse oval and biliary epithelial cells. Expression on newly formed hepatocytes. Differentiation 1990, 45:29-37

32. Evarts RP, Nagy P, Nakatsukasa H, Marsden E, Thorgeirsson SS: In vivo differentiation of rat liver oval cells into hepatocytes. Cancer Res 1989, 49:1541-1547

33. Ahn S, Joyner AL: Dynamic changes in the response of cells to positive hedgehog signaling during mouse limb patterning. Cell 2004, 118:505-516

34. Danielian PS, Muccino D, Rowitch DH, Michael SK, McMahon AP: Modification of gene activity in mouse embryos in utero by a tamoxifen-inducible form of Cre recombinase. Curr Biol 1998, 8: $1323-1326$

35. McCright B, Lozier J, Gridley T: A mouse model of Alagille syndrome: Notch2 as a genetic modifier of Jag1 haploinsufficiency. Development 2002, 129:1075-1082

36. Zong Y, Panikkar A, Xu J, Antoniou A, Raynaud P, Lemaigre F, Stanger BZ: Notch signaling controls liver development by regulating biliary differentiation. Development 2009, 136:1727-1739

37. Murtaugh LC, Stanger BZ, Kwan KM, Melton DA: Notch signaling controls multiple steps of pancreatic differentiation. Proc Natl Acad Sci USA 2003, 100:14920-14925

38. Imayoshi I, Shimogori T, Ohtsuka T, Kageyama R: Hes genes and neurogenin regulate non-neural versus neural fate specification in the dorsal telencephalic midline. Development 2008, 135:2531-2541 